\title{
HISTORIA DE TRES CIUDADES: AH KIN PECH, ACANMUL Y SAN FRANCISCO DE CAMPECHE ${ }^{1}$
}

\author{
Lorraine A. WILLIAMS-BeCK \\ Universidad Autónoma de Campeche \\ Edmundo LOPEZ DE LA ROSA \\ Facultad de Economía, UNAM
}

Era el mejor y el peor de los tiempos; era la edad de la sabiduría y de la estupidez; la época de la fe y de la incredulidad; la estación de la luz y de las tinieblas; era la primavera de la esperanza y el invierno de la desesperación. En el porvenir lo tendríamos todo y no tendríamos nada; todos íbamos directamente al cielo y directamente al lado opuesto. En suma, aquel periodo era tan semejante al actual que las autoridades de más renombre insistían en que la comparación se hiciera solamente en el grado superlativo, tanto en lo bueno como en lo malo (Charles Dickens, Historia de dos ciudades).

Lo que Dickens narra para Europa a fines del siglo XIX, $i$ son hechos históricos o forman parte de una leyenda? A menudo la información de las fuentes nos plantea esta inquietud, amén de lo escrito sobre las ciudades prehispánica y colonial de Campeche. Por más que leemos y revisamos los relatos de cronistas españoles al llegar a esta tierra, no nos despejan la duda, ni separan la leyenda de lo que realmente vieron aquellos hombres al desembarcar en ese lugar mítico: Ah Kin Pech. Pa-

' El presente ensayo cobra mayor claridad por los comentarios que hicieron los colegas José Alberto Abud Flores, José Manuel Alcocer Bernes, Joaquín Lanz y Ana Luisa Izquierdo sobre algunos de los aspectos históricos tratados. Sin embargo, la forma en que aquellos señalamientos aparecen aquí es responsabilidad de los autores. Quisiéramos agradecer también las finas atenciones del personal del Instituto Chiapaneco de Cultura, bajo la dirección en ese entonces del doctor Andrés Fábrigas Puig, y el Departamento de Patrimonio Cultural e Investigación, bajo la dirección en ese entonces del antropólogo Jesús Morales Bermudes, por habernos extendido una cordial invitación para participar en el V Foro de Arqueología de Chiapas en noviembre de 1994, con una versión preliminar del presente ensayo como ponencia. 


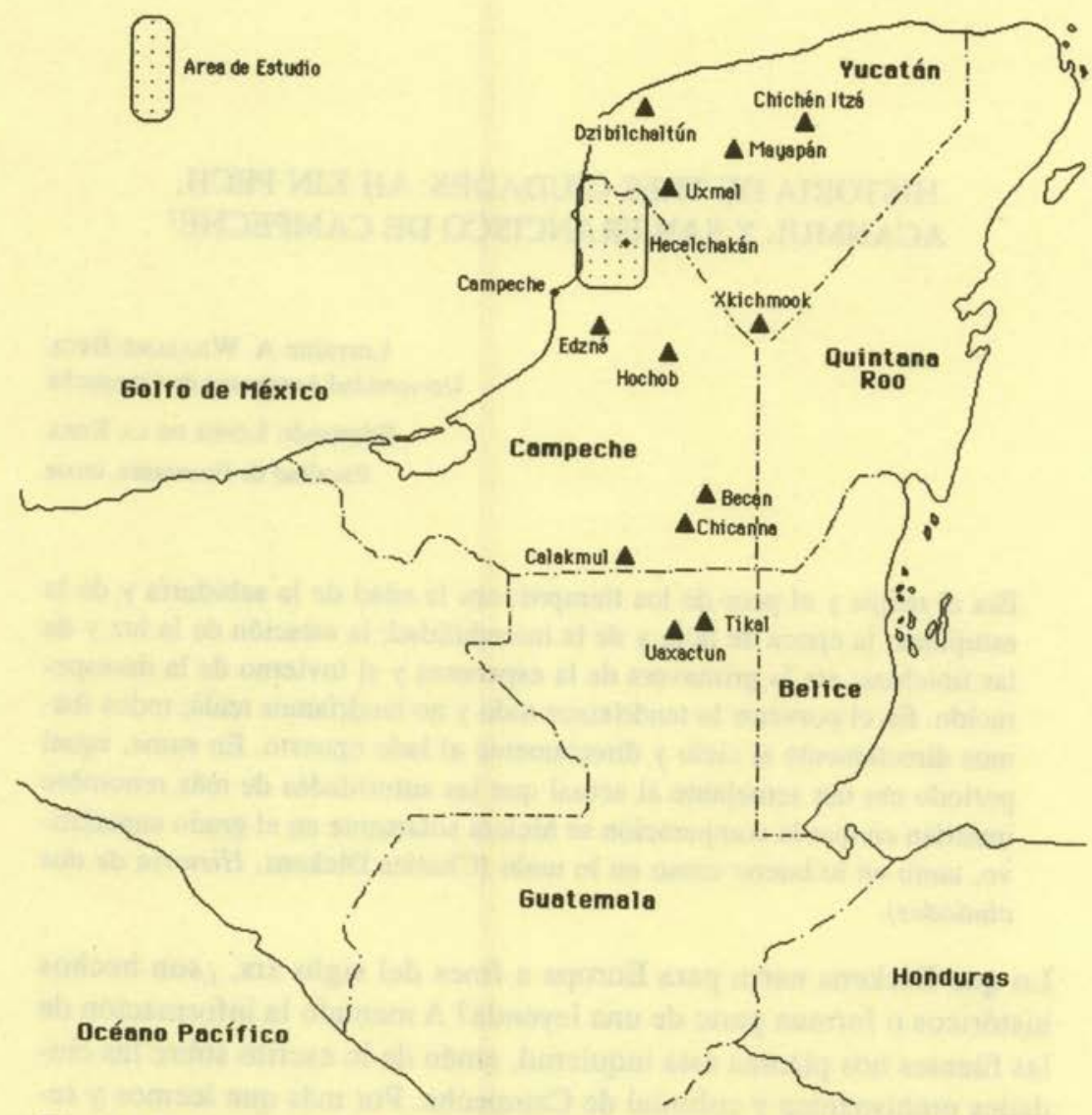

Figura 1. Territorio ỳ poder. Estudio y análisis regional de la zona Camino Real Alto (Provincia Ah Canul)

rece tan débil esa línea divisoria entre leyenda e historia que, cuanto más afirme el consenso general que la actual ciudad capital se asienta encima de templos y edificios monumentales de Ah Kin Pech, la evidencia arqueológica no lo sustenta. ${ }^{2}$

${ }^{2}$ Sólo hay que recordar obras públicas de cableado subterráneo recientes, que, al abrir el corazón del recinto amurallado, no aportaron restos de arquitectura prehispánica monumental, ni serpientes monolíticas o abundantes cantidades de cerámica mundana de la época precontacto. 
Lo mismo sucede con la memoria histórica inmediata. Si revisáramos la información disponible sobre Campeche publicada a principios de este siglo, veríamos que a partir de este momento comienza un relato que cuenta la fundación de esta ciudad. Quizá en el afán de crear una identidad cultural propia a un estado con apenas 10 lustros de vida, esta historia "oficial" no sólo identifica a la ciudad colonial de Campeche con sus raíces mayas, sino también la ubica en el sitio de la ciudad Ah Kin Pech, lugar legendario de sus antepasados. Por ello, es fundamental localizar aquellas ciudades y pueblos autóctonos mencionados en los documentos indígenas, religiosos o militares, ${ }^{3}$ para aclarar la duda.

$\mathrm{Al}$ revisar la información histórica disponible para el área alrededor de la ciudad colonial de Campeche, utilizamos las crónicas Historia verdadera de la Conquista de la Nueva España (Díaz del Castillo, 1632) como fuente primaria, así como Historia de la conquista de Yucatán (Chamberlain, 1982) y el Catálogo de documentos para la historia de Yucatán y Campeche (Pérez Martínez, 1943), como fuentes secundarias detalladamente documentadas. ${ }^{4}$ Agregamos a esta información tres mapas publicados en la Historia cartográfica de la península de Yucatán (Antochiw, 1994) y otros del Archivo General de la Nación.

${ }^{3}$ Este trabajo forma parte del proyecto de investigación "Territorio y Poder: Estudio y Análisis Regional de la Zona Camino Real Alto (Provincia Ah Canul) en Campeche", que ha sido patrocinado por la Universidad Autónoma de Campeche, así como por un generoso apoyo financiero de la Dirección General de Investigación Científica y Superación Académica de la SEP (a través del convenio núm. 93-02-04-001-189; véase figura 1). A ambas instituciones y al USDA Forest Service Southern Region les damos las más cumplidas gracias por su decidido respaldo a nuestros esfuerzos. Una vertiente de este proyecto arqueológico mayor en proceso es promover un análisis bien documentado sobre algunas formas de organización político-territorial, distribución de la autoridad, transmisión del poder, tipos de residencia de familias gobernantes y la localización de sitios mencionados en las crónicas etnohistóricas desde el periodo Clásico hasta el contacto español (del 550 al 1517 de nuestra era).

${ }^{4} \mathrm{Al}$ revalorizar otros tratados históricos, estos sólo retoman lo anteriormente escrito, como son los ejemplos de Francisco López de Gómara (Historia general de las Indias, 1552), quien nunca pisó la tierra firme del Nuevo Mundo; de fray Juan de Torquemada, que copió en su Monarquía indiana (tomo I, libro IV, capítulo III) lo que escribe López de Gómara; y de Diego López de Cogolludo (Historia de Yucatán, 1688), quien asienta lo que Díaz del Castillo apunta. A nuestro parecer, el comentario sobre la fundación de Campeche de fray Diego de Landa (Relación de las cosas de Yucatán, 1560) es muy general, y la documentación y experiencias propias que dispone Gonzalo Fernández de Oviedo no logran la riqueza de Díaz del Castillo y Las Casas para esta zona (Sotelo Regil, 1963: 62-64; Lewis Hanke, Las Casas, historiador, citado en Sotelo Regil, 1963). El Catálogo de documentos para la historia de Yucatán y Campeche, recopilados por Héctor Pérez Martínez en 1943, tampoco vislumbra mayores fuentes de información sobre esta época. 
Confiamos en que los informes de exploradores y conquistadores arriba señalados resaltan factores geográficos específicos. Sin embargo, no deja de llamar la atención que, fuera de aquellos textos clásicos, poco o nada disponemos de otros escritos para esta zona. Cogolludo se quejaba de esta situación también cuando menciona las limitaciones que enfrentó para escribir sobre la conquista de Yucatán y fundación de la Villa de Campeche:

Digo otra vez... que sería posible algún día pese a los interesados; rogando he estado a todos me den los escritos de sus ascendentes, a muchos no se les ha dado más, que si no importara su gloria el quedar aquí escritos, de que yo no tengo omisión voluntaria, como ni en decir lo poco que diré de la fundación de esta villa. A toda ella pongo por testigo, como fú́ este año de mil y seiscientos y cincuenta y cinco personalmente, para haber de sacar esto en limpio del borrador, y poder escribir su fundación, como la de la ciudad de Mérida y la de la villa de Valladolid, porque aunque lo hubiera solicitado por muchas encomiendas, no tenían razón de ella, y sin más de la que fuí, me hube de volver, porque ni aun los papeles antiguos de los archivos para que yo los trabajase y mirase, se me dieron. Y así digo solamente, que por el auto de fundación de la ciudad de Mérida consta, que allí se fundó una villa con el nombre de San Francisco de Campeche, y fue el año de quinientos y cuarenta o el cuarenta y uno, y tengo por más cierto el de cuarenta, pues fue lo primero que poblaron en saliendo de Champotón, y de allí se dice, cómo su iglesia se edificó con titular de Nuestra Señora de la Concepción. Por este auto y por la instrucción del Adelantado dada a su hijo, consta claramente haber errado el bachiller Valencia en su relación, diciendo, que el año treinta y nueve estaba ya poblada esta villa (Cogolludo, 1954 [T]: 254).

¿Qué significaría esto? En todo tiempo y lugar "la recuperación del pasado, antes que científica, ha sido primordialmente política: una incorporación intencionada y selectiva del pasado lejano e inmediato, adecuada a los intereses del presente para juntos modelarlo y obrar sobre el porvenir" (Florescano, 1982: 93). En el relato de López de Cogolludo efectivamente hay una incorporación selectiva del pasado lejano e inmediato, ya que él evita mencionar la pugna de intereses eclesiásticos y económicos que existía entre la orden franciscana, de la cual él formaba parte, y los encomenderos asentados en algunas partes de la península yucateca. Por las acciones de evangelización emprendidas por los frailes, los encomenderos quedaban económicamente afectados por los cambios y reducciones de poblaciones indígenas (González Cicero, 1978: 180-208, esp. 195-199). Por ejemplo, durante el gobierno 
del alcalde mayor de Yucatán Gaspar Gonzales de Ávila, González Cicero ofrece una interpretación sobre un conflicto de esta naturaleza, ${ }^{5}$ con respecto a una instrucción recibida del virrey Mendoza sobre la carga de los tlamemes,${ }^{6}$ donde se especificaba el pago de este trabajo, la carga máxima, las jornadas semanales y el jornal diario.

Debido a este tipo intencional de "revisionismo histórico", algunos autores opinan que "[e]l estudio del Pasado (sic) no es indispensable sino al servicio del presente" (Pereyra, 1983: 17, citando a Chesnaux). A menudo este hecho llega a tal grado que "[c]uando se disuelve por completo la lógica propia del discurso histórico en los zigzagueos de la opción política inmediata, entonces no pueden extrañar ocultamientos, silencios y deformaciones... [sic]" (Pereyra, 1983: 17).

Parece ser sintomático el "ocultamiento" de información sobre los orígenes de Campeche desde la misma época colonial incluso. Cogolludo sufrió - al igual que sufrimos otros interesados - por la falta de acceso a la memoria histórica de Campeche. ¿Por qué el contenido de los documentos de la época nos hace pensar en que parecen haber hecho tabla rasa del registro histórico del Canpech prehispánico?

\section{Las ciudades Ah Kin Pech, San Francisco de Campeche y Acanmul}

En las primeras páginas de su Verdadera historia de la Conquista de la Nueva España, Bernal Díaz del Castillo relata la trágica expedición en 1517 de Hernández de Córdoba, de la que él formó parte. Nos dice este testigo presencial que, una vez superado el conflicto en Cabo Catoche, avanzaron por toda la costa de la península hasta llegar a un punto donde, desde los navíos, vieron a

... un pueblo, y al parecer algo grande, y había cerca de él gran ensenada y bahía; creímos que había río o arroyo donde pudiésemos tomar agua, porque teníamos gran falta della; acabábase la de las pilas y vasijas que traíamos, que no venían bien reparadas; que, como nuestra armada era de hom-

${ }^{5}$ Así dice el texto: "Por la defensa que hacían los franciscanos de los indios, los encomenderos acusaban a los frailes de que ellos cometían también innumerables abusos con los naturales en la construcción de iglesias y monasterios; les reprochaban sus errores, y por tanto les ponían obstáculos en la evangelización e intentaban que no se les diese limosna para sostenerse. Los frailes, a su vez, condenaban públicamente los abusos que cometían los encomenderos y amenazaban con no absolver en confesión a aquellos que abusaban de los indígenas" (González Cicero, 1978: 35-36). 
bres pobres, no teníamos dinero cuanto convenía para comprar buenas pipas; faltó el agua y hubimos de saltar en tierra junto al pueblo, fue un domingo de Lázaro, y a esta causa le pusimos este nombre, aunque supusimos que por otro nombre propio de los indios se dice Campeche [sic].

Como podemos observar (figura 2), es claro que Díaz del Castillo no describe a la actual capital de Campeche, ${ }^{7}$ sino a una zona alrededor del sitio arqueológico de El Cuyo, ubicado inmediatamente al norte de una bahía, frente a una ensenada y cerca del río Hontún. ${ }^{8}$ Luego, la crónica puntualiza otra referencia geográfica:

Y porque en aquellos ancones y bahías mengua mucho la mar, y por esta causa dejamos los navíos anclados más de una legua de tierra y fuimos a desembarcar cerca del pueblo, que estaba allí un buen pozo de buena agua, donde los naturales de aquella población bebían y se servían de él, porque en aquellas tierras, según hemos visto, no hay ríos; y sacamos las pipas para las henchir de agua y volvernos a los navíos... (Díaz del Castillo, 1991: 9; el subrayado es del autor).

En este lugar, ahora tapizado de manglares y manantiales, el nivel del mar es mínimo. De hecho, una vez que baja la marea hay áreas en donde no puede circular lancha alguna. También es impresionante mirar cómo hoy en día todavía podemos ver ojos de agua dulce que brotan como remolinos entre el agua de mar.

Además de esto hay tres mapas antiguos del área ${ }^{9}$ que sugieren la presencia de dos lugares: Lázaro y la recién fundada villa española de San Francisco de Campeche. En el último de ellos (figura 3), aparece una construcción al lado de la costa occidental de la península que indica una ciudad y el nombre de Cápeché y en seguida otro símbolo de ciudad con el nombre de Lázaro (Antochiw, 1994: 120).

${ }^{6} \mathrm{De}$ acuerdo con el Diccionario de la lengua náhuatl o mexicana (1988: 614), el término tlameme se refiere a un individuo que lleva fardos sobre las espaldas, es decir, un cargador.

${ }^{7}$ La ciudad de Campeche se encuentra frente a la bahía.

${ }^{8}$ El río Hontún es una corriente temporal que parece ser alimentado no sólo por manantiales abundantes en la zona, sino por el desagüe natural de tierra adentro durante la temporada de lluvias. Al momento del arribo de los españoles y por ser época de secas, posiblemente lo vieron sin caudal.

${ }^{9}$ Éstos son la Carta del Seno Mejicano, Tierra Firme y América del N. sobre el Atlántico, hasta los $44^{\circ}$ N., Totius Orbis descriptio (1542), del cartógrafo español Sebastián de Santa Cruz, quien de 1526 a 1530 acompañaba a Sebastián Cabot, y Carta de la Península de Yucatán y el Golfo de Honduras (ca. 1645) (Antochiw, 1994: 120). 


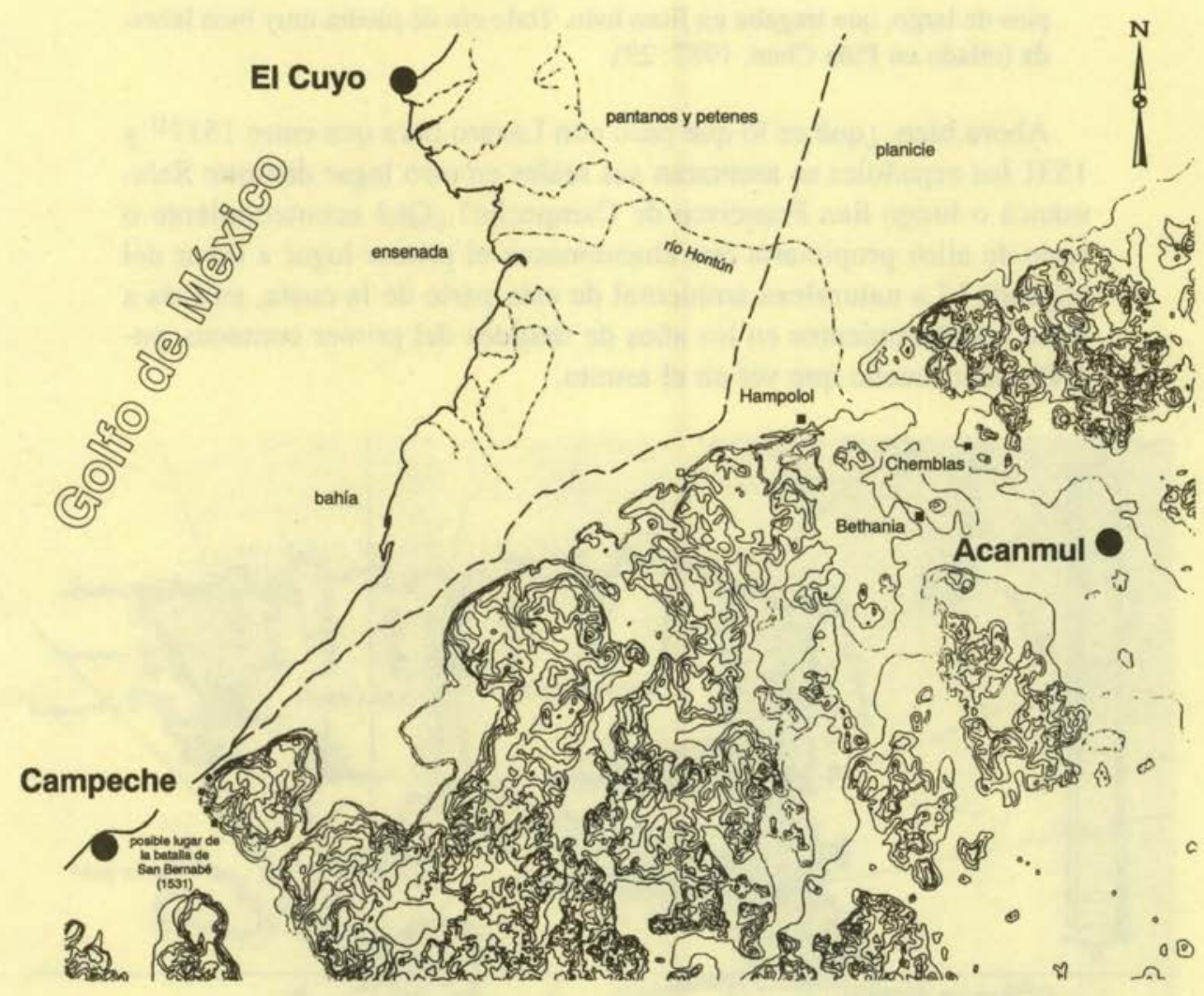

Figura 2

Sobre la identificación de Lázaro, lugar que creemos está cerca del sitio arqueológico El Cuyo, veamos ahora lo que nos cuenta de segunda mano fray Bartolomé de Las Casas: ${ }^{10}$

... a través de un velo de brumas se distinguió la línea luminosa de la costa y a medida que se acercaron fueron viendo el caserío: unas tres mil casas y una vegetación rica y exuberante. Así aparecía desde el mar, pero cuando se fueron acercando, vieron un adoratorio de cal y canto con una torre cuadrada de cantería muy blanqueada, con gradas y, en la pared, figuras de serpientes y otras alimañas. En el fondo del altar había un ídolo con dos leones grandes, salpicados de sangre y más abajo una serpiente de cuarenta

${ }^{10}$ Las Casas no fue testigo presencial de los hechos, sino que retoma lo descrito por otros exploradores y conquistadores. 
pies de largo, que tragaba un fiero león. Todo era de piedra muy bien labrada (citado en Piña Chan, 1987: 29).

Ahora bien, ¿qué es lo que pasó con Lázaro para que entre $1517^{11}$ y 1531 los españoles se asentaran sus reales en otro lugar distinto: Salamanca o luego San Francisco de Campeche? ¿Qué acontecimiento o serie de ellos propiciaría que abandonasen el primer lugar a favor del segundo? La naturaleza ambiental de esta parte de la costa, aunada a otros acontecimientos en los años de después del primer contacto, podrán tener mucho que ver en el asunto.

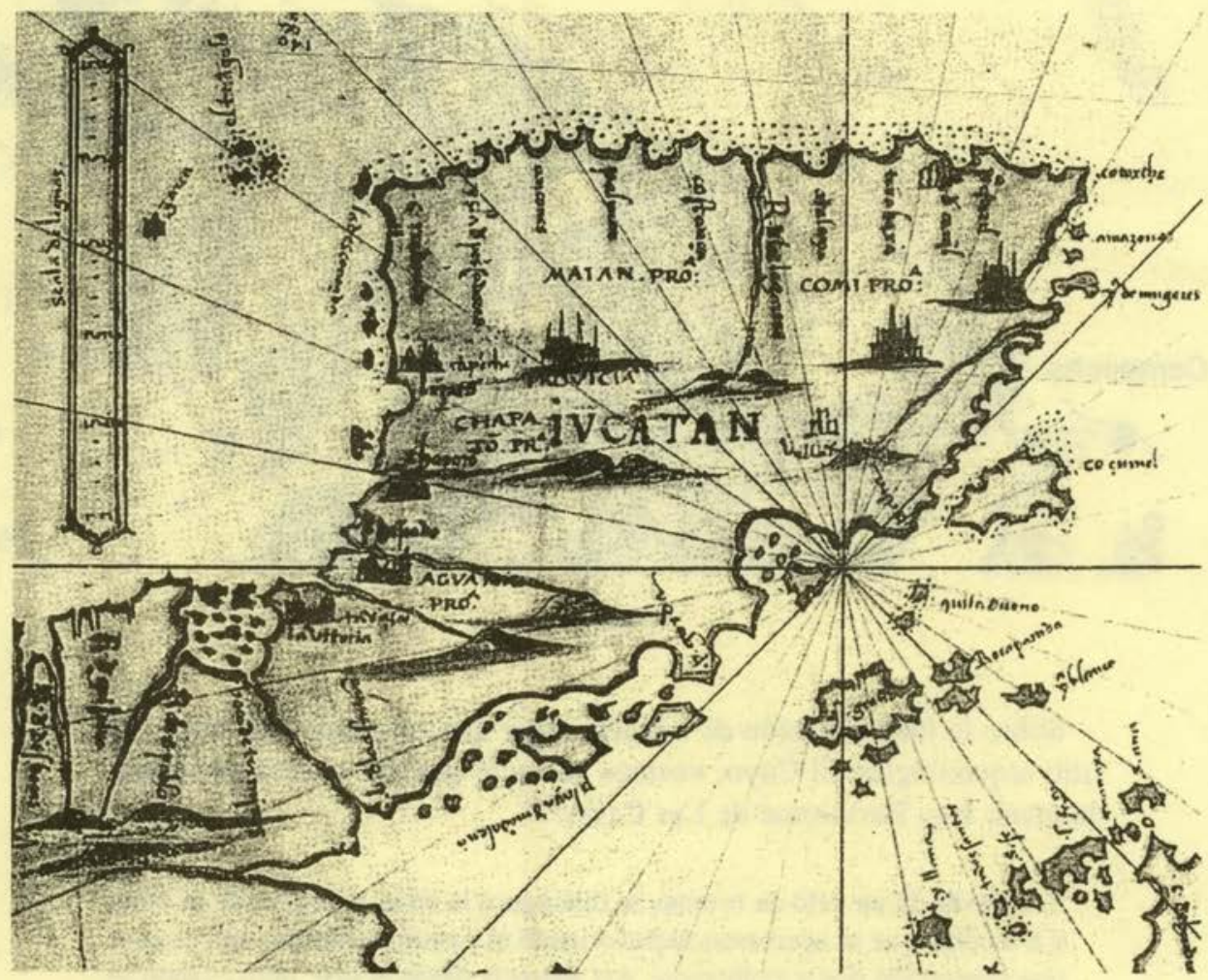

Figura 3. Carta de la Península de Yucatán y del Golfo de Honduras (ca. 1645). Redibujado de Antochiw, 1994, núm. 27

${ }^{11}$ De hecho, la última mención que encontramos en los documentos históricos sobre la ubicación aproximada del lugar de Lázaro viene del Itinerario de la Armada del Rey Católico á la Isla de Yucatan, en la India, el Año de 1518, relato escrito por el comandante y capitán general Juan de Grijalva (García Icazbalceta, 1980, 1: 292). 
En primer lugar, hay una gran diferencia entre el terreno de lo que podría ser el lugar de Lázaro, rodeado de manglares en tierras bajas y fangosas, y lo que sería el asentamiento de Salamanca de Campeche, en donde hay una gran planicie rodeada por colinas en sus costados sur, oriente y poniente, con el lado norte delimitado por el mar. Desde el punto de vista militar español del siglo xvI (cf. Farriss, 1984; Jones, 1987), no cabe duda alguna de que este último lugar, ganado a capa y espada, llenaría los cuadros de seguridad requeridos para un asentamiento colonial, por ser más protegido y asimismo defendible ante cualquier ataque que se le emprendiera.

Después de que los españoles tuvieron su primer contacto con tierras campechanas en 1517, Chamberlain (1974: 133) menciona que

...los mayas de [las Provincias] Canpech y Ah Canul, que al principio parecían no haber demostrado señales activas de hospitalidad, sino que completamente al contrario en realidad habían aceptado quietamente el arribo de los españoles, se fueron haciendo más y más descontentos y obstinados, conforme el yugo español se establecía aún más firmemente sobre sus hombros.

Llegó esta antipatía a grado tal que los pueblos de la Provincia Ah Canul y de Sotuta, bajo el líder Nachí Cocom, sumarían esfuerzos y atacaron al asentamiento español durante una batalla el día de San Bernabé. ${ }^{12}$ Comenta otra vez Chamberlain (1974: 34) que "después de lanzar grandes peñas desde los cerros sobre la población, barrieron impetuosamente por abajo sobre el puñado de defensores. Siguió una de las batallas más feroces y célebres de toda la conquista".

De la probanza de Pedro Álvarez (1543) Chamberlain (1974: 134) narra que

...estando seguros los yndios naturales de la provincia $\left[\right.$ Acanul] ${ }^{13} \mathrm{e}$ de todas las provincias comarcanas, dieron de guerra sobre la que estaba poblada en Campeche, y fue tanta cantidad de yndios ${ }^{14}$ que si Dios milagrosamente no ayudara a los españoles que allí se hallaron, todos mueran a causa de no ser más de quarenta y cinco españoles, e los más de ellos enfermos e dolientes; a la qual dicha batalla e rebolto el dicho Pedro Alvarez salió con una espada e una rodela, hiriendo e matando a los enemigos, y se metió en la más

${ }^{12}$ Este conflicto tuvo lugar el 11 de junio de 1531 .

${ }^{13}$ De acuerdo con una revisión del documento original hecho por Tsubasa Okoshi Harada (comunicación personal, 1995), Chamberlain parece haberse equivocado en la lectura paleográfica de esta palabra, que en su texto se lee como Acamil.

${ }^{14}$ Dice Blas Gonzales que fueron más de 20000 naturales. 
fuerça de ellos e... subió por una sierra donde los dichos yndios estaban hechos fuertes, así de armas como de fuegos para defender a los christianos; el dicho Pedro Alvarez animosamente subió entre ellos, encima de la dicha fuerça, y estaba allí batallando con ellos más de dos horas, con muy grande peligro, hasta tanto que el Gobernador, e tres o quatro de a caballo le guarecieron; y así por el buen esfuerzo del dicho Pedro Alvarez, así como por la buena diligencia de su Gobernador e Capitán General, e los demás que en la dicha batalla se hallaron, se hubo victoria contra los enemigos de nuestra Santa Fe Cathólica, que fue día de San Bernabé, y en la memoria de haberse hallado los christianos en tanto peligro y haber alcanzado tan grande victoria, juraron cada un año, día de San Bernabé, sacar su pendón en procesión, dando gracias a Dios por la merced que aquel día les hizo, la cual dicha memoria está en usanza desta dicha ciudad e hoy día se guarda... [sic].

Las coincidencias entre los nombres Acanul ${ }^{15}$ y Acanmul, el sitio prehispánico que describiremos a continuación, así como el lugar de la batalla ${ }^{16}$ rodeado de colinas, y Salamanca de Campeche, parecen convertirse en hechos que comienzan a traspasar las fronteras entre leyenda e historia.

No obstante, habría que preguntarnos que, salvo la gracia de Dios, ¿cuáles serían las reglas de guerra entre los mayas de la época prehispánica, pues cómo es posible que no mataran a todos los que allí se encontraron si tenían muchos más reclutas que los españoles? Dice Chamberlain (1974: 134) que

...[s]iguiendo su táctica tradicional, los mayas trataron de matar o capturar a Montejo, porque entre ellos la muerte o captura del caudillo de sus adversarios conducía a la victoria. Creían que los españoles abandonarían Yucatán si el Adelantado ya no los mandaba más. Consecuentemente, el mismo Montejo era el punto convergente de los más fuertes combates...

Después de que los mayas no lograron su objetivo principal, Montejo "...parece haber concertado alianzas más o menos permanentes con los caciques que habían sido sus enemigos recientemente" (Chamberlain, 1974: 136).

Ahora bien, ¿qué es lo que le habrá sucedido al asentamiento prehispánico de El Cuyo? La siguiente nota de un encomendero en 1570 nos podría dar algunas pistas que investigar:

${ }^{15}$ Véase lo dicho en la nota 9.

${ }^{16}$ Este lugar parece estar cerca del barrio 7 de agosto en la ciudad capital de Campeche. 
...Tomás López, Oydor de su magestad... mandó despoblar los dichos pueblos para allegarlos junto a los monasterios que tengo dicho, a los pueblos que no querían despoblarse de sus asientos les mandaba poner fuego a las casas y desque se fue desta tierra dexo poder a los religiosos frailes de San Francisco que los pueblos que quisiesen mudallos a los monasterios e que los mudasen y lo que no los quemasen... fue parte para mudarse y que moriese tanta gente como se ha muerto, porque dizen los yndios que unos los mandaban mudar por fuerza y les quemaban sus casas y los cortavan los árboles de frutas que tenían les daba tan gran tristeza en el corazón que se morían... [sic ]. ${ }^{17}$

Además de lo anteriormente expuesto, si revisamos el registro histórico sobre la fundación de otras villas coloniales en la zona del Caribe y en el sureste de la Nueva España, esto nos brinda una nueva interpretación. Los casos particulares de Santo Domingo en la Española y Santa María la Victoria en Tabasco presentan una dinámica parecida a la de Campeche. Al principio ambos lugares se asentaron en un sitio que, años más tarde y por diversas razones todavía no claras, fue cambiado por otro. ${ }^{18}$ Así que el haber trasladado el sitio de Lázaro a una nueva morada, que hoy conocemos como San Francisco de Campeche, tiene antecedentes que parecen indicar que una de las opciones de los españoles era ubicar las villas coloniales en lugares distintos a los que habían sido su primera entrada a tierra firme.

Ahora bien, si El Cuyo pudo haber sido un lugar próximo al de Lázaro en las crónicas y los mapas coloniales, la siguiente interrogante es: ¿en dónde estarían las grandes casas que describe Díaz del Castillo? Su crónica es clara:

...Ya que estaban llenas [las pipas de agua] y nos queríamos embarcar, vinieron del pueblo obra de cincuenta indios con buenas mantas de algodón, y de paz, y a lo que parecía debían ser caciques, y nos decían por señas que

${ }^{17}$ Esta cita es un fragmento retomado de la cédula de museografía del Museo Regional de Campeche, cuyo autor desconocemos.

${ }^{18}$ En el primer caso, el sitio de Santo Domingo fue trasladado a una planicie frente al lugar en donde desembarcaron los españoles, para poder acomodar el trazo urbano y plan de desarrollo comunitario (cf. Veloz Maggiolo y Ortega, 1992). Con respecto a Santa María la Victoria, los documentos narran que fue fundada en un sitio prehispánico, escogido por Montejo el Adelantado para trazar la primera aldea colonial. Ubicado en la orilla del río Grijalva y en medio de una zona pantanosa, quizá en la época de contacto aquel sitio prehispánico y colonial estaba rodeado por campos de cultivo de cacao. Años más tarde y por razones todavía no claras, algunos pobladores solicitaron trasladarse más tierra adentro al sitio de San Juan Bautista de Villahermosa, capital actual del estado de Tabasco (cf. Izquierdo, 1995: 35-39). 
qué buscábamos, y les dimos a entender que tomar agua e irnos luego a los navíos, y señalaron con la mano que si veníamos de hacia donde sale el sol y decían "Castilan, Castilan". Y después de estas pláticas que dicho tengo, nos dijeron por señas que fuésemos con ellos a su pueblo, y estuvimos tomando consejo si iríamos. Acordamos con buen concierto de ir muy sobre aviso, y lleváronnos a unas casas muy grandes, que eran adoratorios de sus ídolos y estaban muy bien labradas de cal y canto, y tenían figurados en unas paredes muchos bultos de serpientes y culebras y otras pinturas de ídolos, y alrededor de uno como altar, lleno de gotas de sangre muy fresca; y a otra parte de los ídolos tenían unas señales como a manera de cruces, pintados de otros bultos de indios; de todo lo cual nos admiramos, como cosa nunca vista ni oída... [ sic ][Díaz del Castillo, 1991: 9; el subrayado es del autor].

Creemos que al lugar con arquitectura monumental a que hace referencia Bernal Díaz del Castillo es Acanmul. De hecho, él mismo menciona una diferencia entre el área de grandes edificios y el puerto al escribir que, una vez amenazados de muerte por los principales del lugar,

...acordamos con buen concierto de irnos a la costa; y así, comenzamos a caminar por la playa adelante hasta llegar enfrente de un peñol que está en la mar, y los bateles y el navío pequeño fueron por la costa tierra a tierra con las pipas de agua y no nos osamos embarcar junto al pueblo que nos habíamos desembarcado, por el gran número de indios que ya se habían juntado, porque tuvimos por cierto que al embarcar nos darían guerra... (Díaz del Castillo, 1991: 10; el subrayado es del autor).

Si hemos interpretado correctamente lo anterior sobre la base que nos brinda una inspección arqueológica y un recorrido en lancha por la costa, todavía persiste un dilema acerca del tiempo que aquellos viajeros estuvieron presentes en estas tierras por vez primera. El consenso general parece indicar una estancia breve. Pero, al revisar documentos eclesiásticos y otras fuentes de la época, encontramos lo siguiente:

Como los españoles desembarcaron en Campeche el domingo de Lázaro y partieron hasta unos días antes de la Semana Santa, está claro que en [esta área] pasaron varios días, inclusive el domingo de Pasión.

La idea de que la permanencia de Hernández de Córdoba en Campeche duró más de tres días, se robustece con otro argumento: cuando los españoles partieron de Campeche, fueron a Champotón, que dista... doce leguas, y a donde llegaron el $1^{\circ}$ de abril de $1517 \ldots$ Los navíos de Colón, de 
Hernández de Córdoba, de Grijalva o de Cortés, navegaban con buen tiempo hasta veinte leguas en un día, según los cronistas, y no se guarda memoria en Campeche o Champotón, de que un velero haya tardado de un punto a otro más de dos días en las circunstancias más azarosas (Sotelo Regil, 1963: 68-69).

Si confiamos en la interpretación temporal del itinerario por Sotelo Regil, no sólo se nos explica el que pudieron haberse ido los 20 kilómetros tierra adentro para conocer Acanmul y sus alrededores, sino que también es la razón de la premura de la subsecuente partida: los españoles ya habían agotado la paciencia de sus anfitriones.

Bosquejo geográfico y arqueológico sobre El Cuyo, San Francisco de Campeche y Acanmul

Ahora volvemos a evaluar la bibliografía especializada para los sitios El Cuyo, Acanmul y la ciudad colonial de Campeche. A dos kilómetros en línea recta hacia el oeste y noroeste del río Hontún, encontramos restos del extenso sitio arqueológico El Cuyo en el mar. Al momento en que la marea baja prácticamente no hay agua, por lo que uno puede caminar entre las piedras que formaron el núcleo de una estructura (Eaton, 1978: 28). A esto se le suman miles de fragmentos de cerámica ${ }^{19}$ que muestran una temporalidad para el sitio desde el Preclásico Tardío hasta el Posclásico Temprano (de 300 años antes de nuestra era hasta 1200 años de nuestra era; Andrews y Corletta, 1995: 105-106; Andrews y Vail, 1990: 42) o, inclusive, hasta más tardío (Ball, 1978: 90-93), lo que parece demostrar que sí existe evidencia arqueológica asociada a una estructura posiblemente descrita por Las Casas. Sin embargo, conviene aclarar que fuera de este núcleo de piedras no vemos más construcciones importantes. Esto seguramente se debe a dos razones en particular: una, que la zona de playa está cubierta por una extensa y densa vegetación de manglar, que prácticamente oculta toda visibilidad de la superficie; y, en seguida, creemos que otras estructuras bien podrían estar bajo el agua (Andrews y Corletta, 1995), ya que en fechas recientes ha habido un incremento en el nivel del mar. La ensenada termina a

${ }^{19}$ La distribución de restos de cerámica en la costa y bajo el agua que observamos en cantidades abundantes durante nuestro recorrido por el lugar, se extiende por lo menos a unos $500 \mathrm{~m}$ al oriente a punta El Cuyo y otros cuantos metros más hacia la desembocadura del río Hontún. Es difícil estimar la extensión hacia tierra adentro, debido a que hay una densa vegetación de manglar en la zona. 
cuatro kilómetros al sur de El Cuyo y de ahí comienza una bahía de más de 10 kilómetros de longitud. En el límite sur de esta bahía, los españoles fundarían la ciudad colonial de Campeche.

Ahora bien, los trabajos arqueológicos efectuados en esta ciudad y puerto (Ruz, 1969: 73; Eaton, 1978: 28; Andrews, 1977: 67, 1983; Burgos, 1986), y más aún, los kilómetros excavados por obras públicas que se realizan en el recinto amurallado (cf. Suárez Aguilar y Ojeda Mas, 1996), indican que no existía un sitio prehispánico importante previo a la ciudad colonial. Recordemos también que los grandes asentamientos prehispánicos en la península de Yucatán por lo general están situados tierra adentro y unidos al mar a través de pueblos costeros o posibles puertos integrados al área con un control directo administrativo de tales ciudades, como son Xelhá para Cobá (Robles, 1979), Champotón para Edzná o Chakanputún (Millet, 1994) e Isla Cerritos para Chichén Itzá.

$\mathrm{Si}$ aceptamos la idea de que "el rango máximo de un directo control administrativo para cualquier centro en una sociedad enlazada por transporte terrestre tendría un radio de aproximadamente $20 \mathrm{~km}$, o un estandarizado 'viaje de alrededor de un día'" (Johnson, 1987: 115; Renfrew, 1975: 14; citados en Kepecs, Boucher y Burton, 1991: 4), ${ }^{20}$ la relación entre punta El Cuyo y Acanmul cumple esta posibilidad, ya que ambos están separados por menos de $20 \mathrm{~km}$. A esto agregaríamos la facilidad de movimiento en el río Hontún, que si lo recorriéramos en temporada de lluvias a $10 \mathrm{~km}$ tierra adentro, poco antes de cruzar el actual pueblo de Chemblás llegaríamos a un extenso bajo que nos deja en la puerta del sitio arqueológico de Acanmul, a unos cinco kilómetros de distancia de éste.

A pesar de que existen referencias sobre Acanmul desde mediados de los años veinte (Quintana Bello desde 1927 hasta 1938; Marquina, 1939: 13), fue hasta la década de 1940 cuando Pollock (1980: 537-541) efectuó un estudio arquitectónico del lugar. En él, Pollock presenta un mapa esquemático de las estructuras, recalcando una descripción breve de nueve de ellas. Partiendo de su croquis como punto de referencia, durante dos semanas y media, en mayo de 1994, hicimos un levantamiento

${ }^{20} \mathrm{Sin}$ embargo, este requisito no lo cumplen otros puertos-ciudades propuestos anteriormente, como lo de Isla Cerritos-Chichén Itzá, en la que Andrews, Gallareta y Cobos (1989: 91-95) presentan una serie de sitios que pudieron ser enlaces entre Chichén Itzá y la costa. Menos problemática es la relación de Champotón-Edzná, que aunque separados por casi 60 kilómetros, existe una red fluvial desde el río Champotón hasta el canal artificial que conecta a éste con el corazón mismo de aquella ciudad prehispánica. 


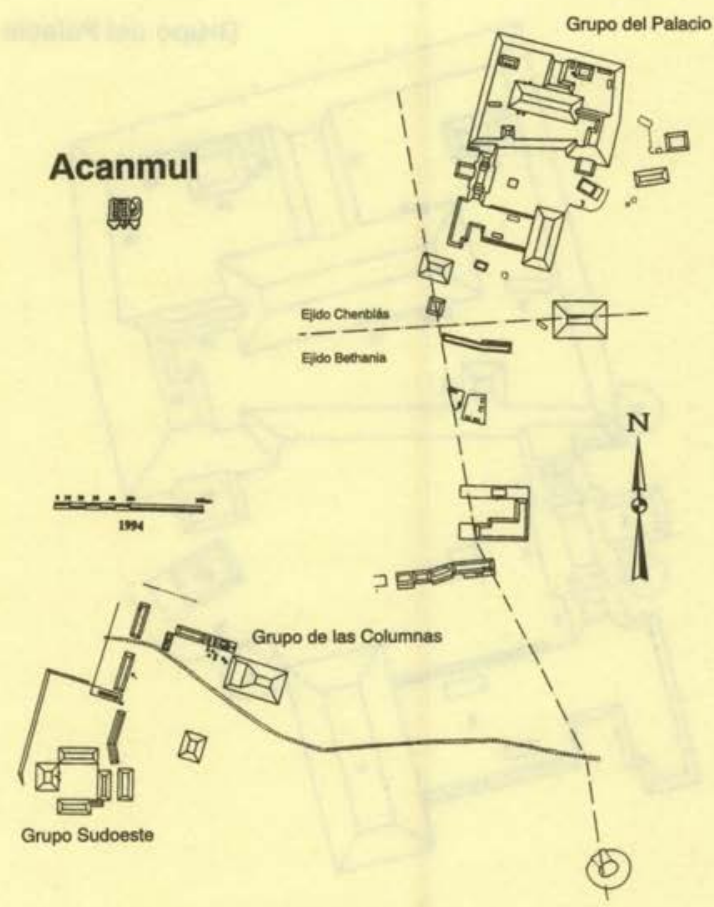

Figura 4. Acanmul

arquitectónico (figura 4) en tres de los cuatro grandes grupos mencionados: el del "Palacio" (según Pollock, 1980), el Conjunto de las Columnas y al que llamaríamos el grupo sudoeste. ${ }^{21}$

El "Palacio" está ubicado en el extremo norte de un conjunto arquitectónico y colocado sobre una gran plataforma subestructural, ${ }^{22}$ encima de la cual hay otros elementos arquitectónicos como estructuras abovedadas, muros de piedra con columnas, montículos y plataformas subestructurales de menor tamaño (figura 5). Pollock observa que el inmueble mayor probablemente contiene varias etapas constructivas. En éste llaman la atención estructuras y aposentos abovedados revestidos con estuco del periodo Clásico, que fueron rellenados con piedra posteriormente, quizá en el periodo Posclásico. En lo que podría ser la última etapa constructiva, esta gran estructura se convertiría en una enorme plataforma subestructural, encima de la cual hay restos de cuartos

${ }^{21}$ Este último grupo arquitectónico no será tratado en el presente ensayo.

${ }^{22} \mathrm{La}$ gran plataforma subestructura mide unos $90 \mathrm{~m}$ por $75 \mathrm{~m}$, con un alzado de aproximadamente $6 \mathrm{~m}$. 


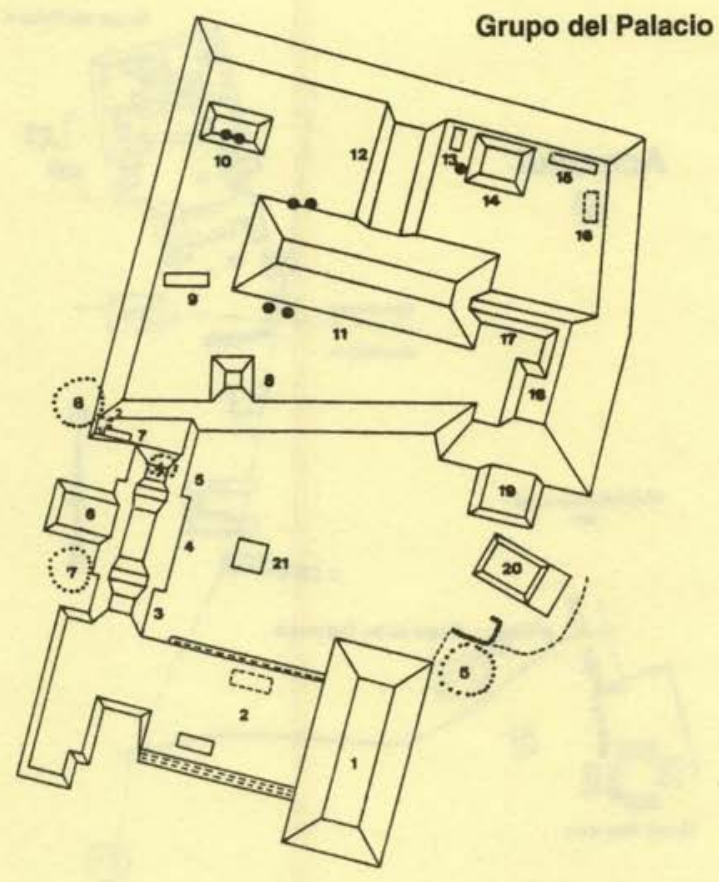

Figura 5. Acanmul. Grupo del palacio

con muros de piedra sin techo de bóveda y columnas en sus fachadas de acceso. Estudios arqueológicos de sitios del Posclásico a lo largo de la costa oriental de la península ${ }^{23}$ y tierra adentro en Quintana Roo $^{24}$ mencionan la presencia de estructuras "tapadas" del periodo Clásico, formando así basamentos piramidales en las cuales levantarían aposentos parecidos a los de Acanmul. Las otras estructuras del conjunto, también del horizonte Clásico, parecen seguir este mismo patrón de modificación por relleno arquitectónico a propósito.

Aproximadamente $250 \mathrm{~m}$ al sudoeste del grupo "Palacio", Pollock hace especial hincapié en las dos principales estructuras que forman parte del Conjunto de las Columnas (figura 6). En ello vemos la estructura 8, que parece haber sido modificada por relleno arquitectónico, rema1975).

${ }^{23}$ Como son el sitio de Xcaret y otros en la Isla de Cozumel (cf. Sabloff y Rathje,

${ }^{24}$ Peter D. Harrison presentó una ponencia en la LX Reunión de la Sociedad Americana de Arqueología en Nueva Orleans, en 1996, en la que describe obras prehispánicas que modifican la arquitectura del periodo Clásico en esta zona, particularmente durante el periodo Posclásico. 


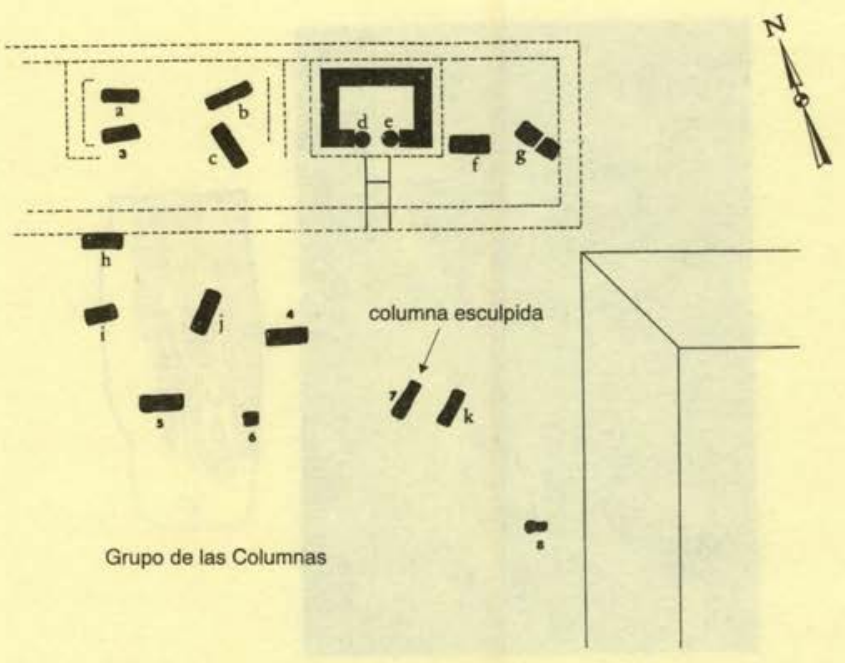

Figura 6. Acanmul. Grupo de las columnas

tando el costado oriental del grupo. La estructura 9, al lado norte del grupo de patio, "es notoria por el gran número de columnas, tanto lisas como esculpidas, a lo largo de su fachada sur" (Pollok, 1980: 540; traducción del inglés por los autores). También llama la atención que en el patio formado por este conjunto hay varias columnas caídas, entre las cuales encontramos un ejemplo esotérico con un individuo esculpido en altorrelieve (figura 7). Éste nos recuerda los motivos iconográficos contemporáneos del horizonte Posclásico Temprano en Chichén Itzá. Unos cuantos fragmentos de cerámica del tipo Chen Mul Modelado de los horizontes Hocabá-Tases (del 1200 al 1450 de nuestra era), recolectados en la superficie, nos sugieren un posible indicador temporal para este conjunto, único en su género a unos 20 kilómetros de la costa campechana del Golfo.

Finalmente, ¿hasta dónde podemos fijar la frontera sur de la provincia Ah Canul, que colinda con la de Canpech en el siglo xvi? Basándose en el códice de Calkiní, Roys (1957: 15-16, 168-169) señala al río Hontún como su límite sudoeste; expresando, sin embargo, que no hay elementos definitivos para fijarlo en tal lado (figura 8). Investigaciones recientes de Okoshi Harada (1992) y Quezada (1993), sobre la base que ofrecen las fuentes coloniales, consideran que los lindes estaban más al norte de lo que Roys pensó (figura 9). Si fuera así, entonces el río Hontún ya no sería una frontera natural entre dos provincias, sino un arroyo de temporal localizado en la 

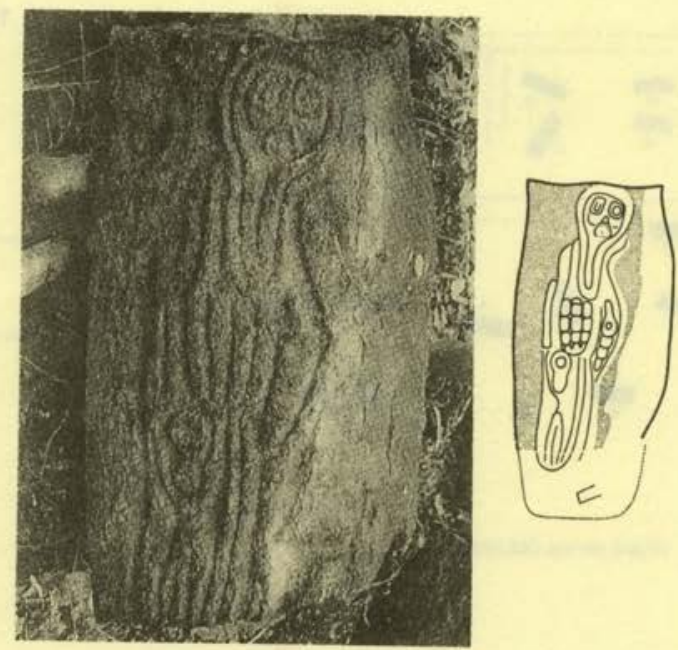

Figura 7. Acanmul. Columna esculpida en altorrelieve

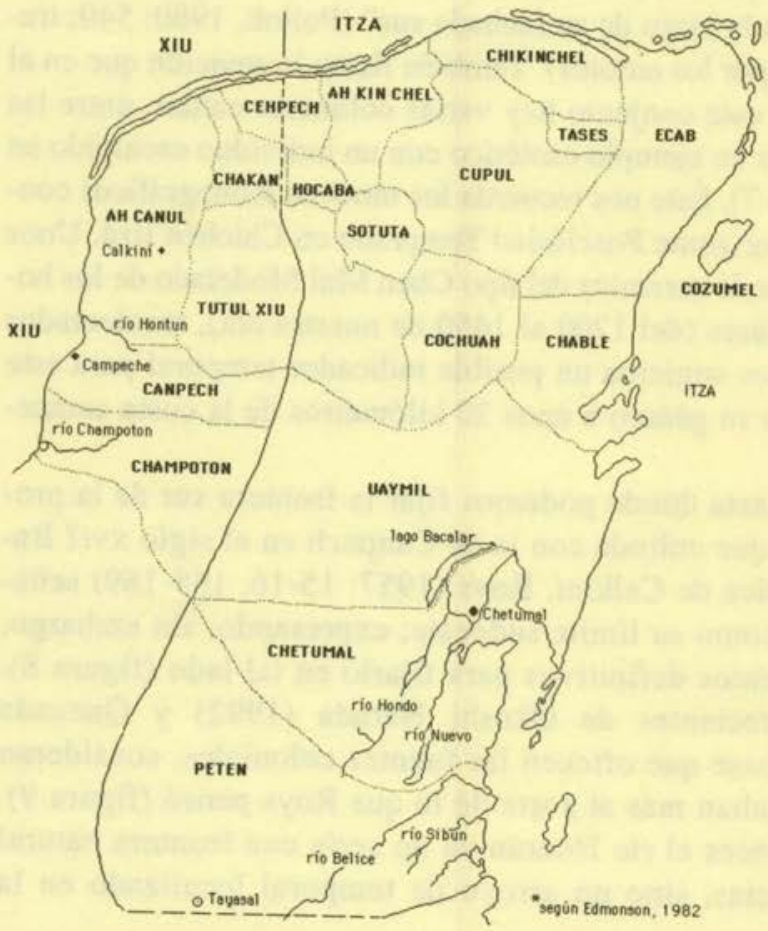

Figura 8. La Península de Yucatán en el siglo XVI

Estudios de Cultura Maya. Vol. XX, 1999 Instituto de Investigaciones Filológicas/ Centro de Estudios Mayas, UNAM ISSN 0185-2574 


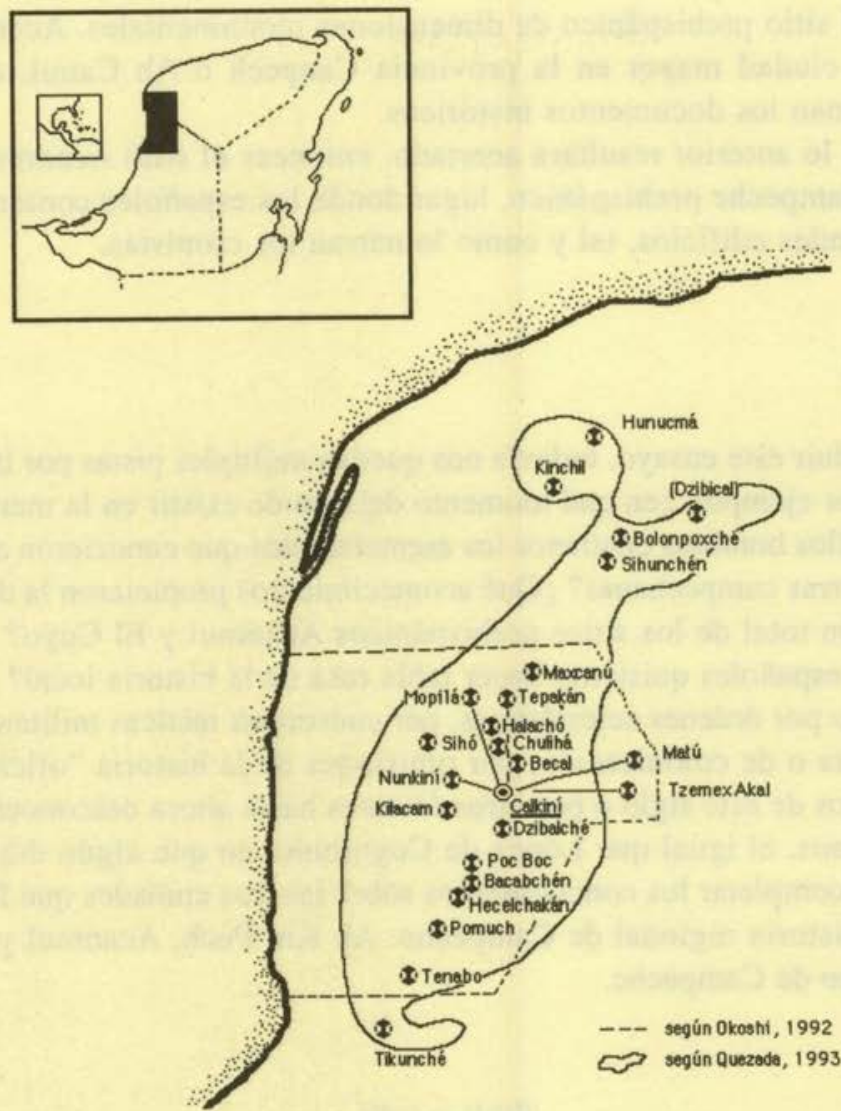

Figura 9. La Provincia de Ah Canul a mediados del siglo xvı

provincia Canpech o Ah Canul que, además, desemboca en una gran ensenada descrita por los cronistas en el siglo Xvi.

Como comentario final y desde aquellos datos que brindan las investigaciones arqueológica e histórica hasta ahora, quisiéramos proponer lo siguiente:

1. El sitio prehispánico El Cuyo es el pueblo que vieron los españoles al llegar a tierras campechanas y cerca a él y junto al río Verde o Hontún bautizarían un lugar con el nombre de Lázaro.

2. El Cuyo es el puerto costero de la gran ciudad tierra adentro, Acanmul. 
3. El sitio prehispánico de dimensiones monumentales, Acanmul, es una ciudad mayor en la provincia Canpech o Ah Canul, como mencionan los documentos históricos.

4. Si lo anterior resultara acertado, entonces el sitio Acanmul sería el Campeche prehispánico, lugar donde los españoles contemplaron grandes edificios, tal y como lo narran los cronistas.

\section{Epílogo}

Al concluir este ensayo, todavía nos quedan múltiples pistas por investigar. Por ejemplo, ¿en qué momento dejaron de existir en la memoria de aquellos hombres cristianos los asentamientos que conocieron al llegar a tierras campechanas? ¿Qué acontecimientos propiciaron la desintegración total de los sitios prehispánicos Acanmul y El Cuyo? ¿Por qué los españoles quisieron hacer tabla rasa de la historia local? ¿Habría sido por órdenes eclesiásticas, por cuestiones tácticas militares de conquista o de colonización, por omisiones de la historia "oficial" a principios de este siglo o por otros factores hasta ahora desconocidos? Confiamos, al igual que López de Cogolludo, en que algún día será posible completar los conocimientos sobre las tres ciudades que forjaron la historia regional de Campeche: Ah Kin Pech, Acanmul y San Francisco de Campeche.

\section{Bibliografía}

ANDREWs, Anthony P.

1977 "Reconocimiento arqueológico de la costa norte del Estado de Campeche", en Boletín de la Escuela de Ciencias Antropológicas de la Universidad de Yucatán, 4 (24): 64-77.

1983 Ancient Maya Salt Production and Trade, University of Arizona Press, Tucson.

ANDREws, Anthony P., y Robert CoRLETTA

1995 "A Brief History of Underwater Archaeology in the Maya Area", en Ancient Mesoamerica, 6: 101-117.

ANDREwS, Anthony P., y Gabriel VAIL

1990 "Cronología de sitios prehispánicos costeros de la Península de Yucatán y Belice", en Boletín de la Escuela de Ciencias Antropológicas de la Universidad de Yucatán, 18 (104-105): 37-66. 
ANDrews, Anthony P., y T. GaLlereta Negrón

1986 "The Isla Cerritos Archeological Project, Yucatán, México", en Mexicon, VIII (3): 44-48.

Andrews, Anthony P., T. Gallereta Negrón, F. Robles Castellanos, R. Cobos Palma y P. Cervera Rivero

1988 "Isla Cerritos: an Itzá Trading Port on the North Coast of Yucatán, México", en National Geographic Research, 4 (2): 196-206.

ANTOCHIw, Michel

1994 Historia cartográfica de la península de Yucatán, CIESAS, Gobierno del estado de Campeche y Grupo Tribasa, México.

BALL, Joseph W.

1978 "Archaeological Pottery of the Yucatan-Campeche Coast", en Eaton y J. W. Ball, Studies in the Archeology of Coastal Yucatan and Campeche, Mexico, Nueva Orleans, Middle American Research Institute Publication, 46.

Burgos V., Rafael

1986 "Salvamento arqueológico en una fortificación colonial de Campeche", en Boletín de la Escuela de Ciencias Antropológicas de la Universidad de Yucatán, 14 (79): 11-18.

Chamberlain, Robert S.

1982 Conquista y colonización de Yucatán: 1517-1550, traducción de Álvaro Domínguez Peón, Editorial Porrúa, México.

DíaZ del CASTILlo, Bernal

1991 Historia verdadera de la Conquista de la Nueva España, Alianza Editorial, México.

DiCKens, Charles

1992 Historia de dos ciudades, Editores Mexicanos Unidos, México.

EATON, Jack D.

1978 "Archaeological Survey of the Yucatan-Campeche Coast", en Eaton y J. W. Ball, Studies in the Archaeology of Coastal Yucatan and Campeche, Mexico, Middle American Research Institute, Nueva Orleans, 46: 1-67.

FARRISS, Nancy

1984 Maya Society under Colonial Rule: The Collective Enterprise of Survival, University of Princeton Press, Princeton.

FloResCANo, Enrique

1983 "De la memoria del poder a la historia como explicación", en E. Florescano (ed.), Historia ¿para qué?, Siglo XXI, México, pp. 91128. 
Garcia ICAZBaLCETA, Joaquín

1980 Colección de documentos para la historia de México, Editorial Porrúa, México.

GonzÁlez Cicero, Stella María

1978 Perspectiva religiosa en Yucatán: 1517-1571, El Colegio de México, México.

IZquIERDo, Ana Luisa

1995 El abandono de Santa María la Victoria y la fundación de San Juan Bautista de Villahermosa, UnAM, Centro de Estudios Mayas del Instituto de Investigaciones Filológicas, México.

JONES, Grant D.

1989 Maya Resistance to Spanish Rule: Time and History of a Colonial Frontier, University of New Mexico Press, Albuquerque.

KePECS, Susan, Sylviane BOUCHER y James BURTON

s.f. "The Itzá in Chikinchel: the Politics of Salt", manuscrito inédito de la ponencia presentada en el 47 Congreso Internacional de Americanistas, Nueva Orleans, 7-11 de julio de 1991.

LANZ, Manuel A.

1905 Compendio de la historia de Campeche, El Fénix de Pablo Llovera Marcin, Campeche.

LAS CASAS, Bartolomé de

1953 Historia de las Indias, 4 volúmenes, Biblioteca de Autores Españoles, Madrid.

LOPEZ De Cogolludo, Diego

1955 Historia de Yucatán, 3 volúmenes, Campeche, Talleres Gráficos del Gobierno del Estado.

MARQUINA, Ignacio

1939 "Introducción", en Instituto Panamericano de Geografía y Estadística, Atlas arqueológico de la República Mexicana, INAH, México.

Millet CAmara, Luis

1994 Mirador campechano, Talleres Gráficos de la Universidad Autónoma de Campeche, Campeche.

OKoshi HaRADA, Tsubasa

1992 Los canules: análisis etnohistórico del códice de Calkiní, tesis de doctorado en antropología, Facultad de Filosofía y Letras, UNAM, México.

Pereyra, Carlos

1983 "Historia ¿para qué?", en E. Florescano (ed.), Historia ¿para qué?, Siglo XXI, México, pp. 9-32. 
Pérez Martínez, Héctor

1943 Catálogo de documentos para la historia de Yucatán y Campeche, Museo Arqueológico, Histórico y Etnográfico de Campeche, Campeche.

PINAa Chan, Román

1987 Campeche durante el periodo colonial, Talleres Gráficos del Gobierno del Estado, Campeche.

Pollock, Harry E. D.

1980 The Puuc: an Architectural Survey of the Hill Country of Yucatan and Northern Campeche, Mexico, Memoirs of the Peabody Museum of Archaeology and Ethnology, vol. 19, Cambridge.

Quezada, Sergio

1993 Pueblos y caciques yucatecos: 1550-1580, El Colegio de México, México.

Quintana Bello, Nazario

1927 Informe sobre la localización de un montículo en Tixmucuy, y la necesidad de proceder a la limpieza de las ruinas de Chenhuhá, Acanmul, Dzibinecac, 26 de febrero de 1927, Archivo Técnico del Departamento de Monumentos Prehispánicos (ATDMP) del INAH, t. III (19), 2 pp., 4 fotos.

1937 Informe que comunica la existencia de la zona arqueológica de El Cuyo, a unos 40 kilómetros de la Isla de Piedra, 31 de agosto de 1937, ATDMP del INAH, t. III (6), 1 p.

1938a Informe de la visita practicada a las zonas arqueológicas de Chulhac, Jaina, Acanmul, Kayal, Xcocha, Xcalumkin y Etzná-Tixmucuy, 31 de diciembre de 1938, ATDMP del INAH, t. CLXVI (5), 1 p., 3 fotos.

1938b "Descubrimiento de Ah-Kin-Pech", en Ah-Kin-Pech, vol. 1 (3).

RoYs, Ralph L.

1957 The Political Geography of Yucatan, Washington, D. C., The Carnegie Institution of Washington, publicación núm. 613.

Ruz LhUILlier, Alberto

1969 "La costa de Campeche en tiempos prehispánicos", en Serie investigaciones, 18 , INAH, México.

SABLOFF, Jeremy A., y William RATHJE

1975 Archaeological Investigations in Cozumel, Quintana Roo, Mexico, University of New Mexico Press Occasional Papers, Albuquerque,

Sotelo ReGIL, Luis F.

1963 Campeche en la Historia, Talleres de la Imprenta Manuel León Sánchez, México. 
SuÁreZ Aguilar, Vicente, y Heber OJeda Mas

1996 Arqueología histórica en la ciudad de Campeche, Universidad Autónoma de Campeche, Campeche.

Veloz Maggiolo, Marcio, y Elpidio Ortega

1992 La fundación de la villa de Santo Domingo, Comisión Dominicana Permanente para la Celebración del Quinto Centenario del Descubrimiento y Evangelización de América, Santo Domingo, República Dominicana.

Williams-Beck, Lorraine A., y Edmundo LÓPEZ DE LA RoSA

s.f. Territorio y poder: Estudio y análisis regional de la zona Camino Real Alto [Provincia Ah Canul], en Campeche. Anteproyecto de Investigación inédito, entregado al Consejo de Arqueología del INAH. 